\title{
Notas preliminares como contribución a los estudios culturales sobre la arquitectura contemporánea costarricense
}

\author{
Carlos Altezor Fuentes
}

Ensayo

Afiliación: Independiente, Montevideo, Uruguay

E-mail: arqaltezor@adinet.com.uy

Recibido:18 de noviembre del 2018

Aceptado: 12 de abril del 2019

\begin{abstract}
Resumen:
Las presentes notas tienen como objetivo exponer de manera breve la situación de la arquitectura costarricense a finales del siglo XX. A su vez, como su evolución ha contribuido de manera significativa al desarrollo de la cultura arquitectónica de nuestro país por medio de su enseñanza en la Escuela de Arquitectura de la Universidad de Costa Rica y los aportes de múltiples profesionales en la disciplina. Finalmente, se destaca el impacto de la literatura sobre arquitectura, patrimonio e identidad costarricense.
\end{abstract}

Palabras clave: arquitectura; Costa Rica; estudios culturales; historia; identidad.

Preliminary notes as a contribution to the Cultural Studies of Costarican contemporary architecture

\begin{abstract}
:
The following notes have the objective to briefly portray the situation of Costarican architecture from the late XX century. Moreover, it contributes significantly to the development of the architectonic culture in our country through the teachings of the Architecture School of the University of Costa Rica and the contributions of multiple professionals within the discipline. Finally, it emphasizes the impact of literature on architecture, heritage and the Costarican identity.
\end{abstract}

Keywords: architecture; Costa Rica; cultural studies, history; identity.

\section{Carlos Altezor Fuentes}

Arquitecto. Ha sido docente e investigador en la Universidad de Costa Rica, en la Universidad de la República del Uruguay, en la Facultad de Arquitectura y urbanismo de la Universidad Central de Venezuela y la Escuela de Ingeniería de Cottbus, Alemania. Autor de Arquitectura urbana en Costa Rica: Exploración Histórica 1900-1950 (1996). Actualmente Investigador honorario en Facultad de Humanidades, Uruguay. 


\section{Introducción}

$\mathrm{C}$

uando decidimos diseñar las presentes notas, recordamos unas líneas escritas para una publicación de la Escuela de Arquitectura de ya hace unos años. Consistían en una opinión sobre nuestra experiencia de trabajo allí en un corto período de enseñanza. Nuestro gran amigo y docente, el arquitecto Jorge Grané, nos brindó esa oportunidad en la edición de la Memoria 25 Aniversario de la Escuela de Arquitectura de la Universidad de Costa Rica 1971-1996. Evoco hoy esas líneas pensando que siguen vigentes para mí, ratificando que mi estadía en la Escuela fue una experiencia inolvidable, que hice muy buenos amigos, que me ayudaron afectuosamente en la didáctica, me alentaron en mi trabajo de investigación y en el logro de una licenciatura que me llena de orgullo. Satisfacción y nostalgia por el lugar y el tiempo que viví.

Pretenden estas notas también a través de informaciones y medidas reflexiones, destacar actitudes de arquitectos, alumnos y profesores, que en el medio nacional, han contribuido a la cultura arquitectónica costarricense. Son la estirpe de una profesión y una disciplina universal que supone estudio, entrega y sacrificio por el mejoramiento del hábitat y del reconocimiento de un arte arquitectónico nacional, haciendo honor a la cultura de Costa Rica. A ellos, atrevidamente, están dedicadas estas líneas, tal vez para alimentar un futuro diálogo constructivo y señalando a continuación algunos tópicos para el debate.

\section{Una visión panorámica de la cultura arquitectónica costarricense desde finales del siglo XX}

Con la intención de referirnos a la cultura arquitectónica nacional del siglo XX, se debe citar como oportunidad nuestra primera investigación sobre la historia de la arquitectura costarricense en el entorno de principios del siglo y la formación de expresiones modernas en su primera mitad. Llevada a cabo en la Escuela de Arquitectura de la Universidad de Costa Rica, posibilitó su publicación por la Editorial Tecnológica en 1985'. Aquel antecedente nutre las actuales líneas de reflexión que se someten al conocimiento, la interpretación y la crítica. Como expresáramos en la introducción de nuestro libro en los años ochenta, que ellas "sirvan como potencial motivación para que otros trabajos de investigadores profundicen temáticas o criterios de análisis sobre los temas aludidos". Y estos deseos, jvaya si se concretaron a todo mérito en los últimos años por investigadores del medio profesional y académico!

1 Arquitectura urbana en Costa Rica. Exploración histórica 1900- 1950 (1986). Editorial Tecnológica de Costa Rica. Ver también la obra de Richard Woodbridge Paris Historia de la Arquitectura en Costa Rica (2003), Editorial Tecnológica de Costa Rica.

$2 \quad$ Nos advierte: "... el concepto de cultura es reexaminado de manera crítica en las CCSS a tal punto que algunos proponen volver al sentido restringido de la palabra, es decir, el que refiere exclusivamente a las producciones intelectuales y artísticas, el concepto experimenta una difusión notable entre las CCSS" (Cuche, 1999, p.145). Este tema - del arquitecto autor y artista- implica sin duda el recurrente tema del papel de las generaciones y del individuo en la historia. Se debe dejar constancia de que no pretendemos pensar la historia de la arquitectura como historia de las generaciones, entendidas como "escuelas" formadas en torno a grandes maestros 0 personalidades que se constituyen en hitos de las historias del arte y del arte mayor: la arquitectura. Tampoco presentar al arquitecto como artista-genio, visión que ha predominado en la historiografía desde siglos atrás y hoy superada.
En primer lugar, un marco de acepciones debe ser contemplado. Aclaraciones sobre el sentido de algunas palabras que usamos, guían nuestro pensamiento y la consiguiente valoración que puedan realizarse sobre los hechos observados de la arquitectura. En la grey de nuestra profesión, aquellas definiciones, conceptos y opiniones no son siempre compartibles y constituyen frecuentemente motivo de polémica; y es bueno que así sea, como una forma de mantener vivo el conocimiento y las reflexiones críticas sobre el motivo de nuestros desvelos.

Participamos en la opinión de que la arquitectura de nuestro territorio contribuye a la cultura general de Costa Rica, enriqueciéndola y difundiéndola en el concierto regional y sudamericano. Entendiendo por cultura no sólo las expresiones superiores del desarrollo científico, intelectual y artístico del país, sino el conjunto de manifestaciones históricas y sociales que a la vez construyen en el tiempo esa cultura y diseñan una particular identidad. Cuando hablamos de cultura arquitectónica, no nos olvidamos que ella comprende aspectos tecnológicos, artísticos, económicos y de raíces socialesantropológicas en el marco urbano y territorial. A pesar de esta afirmación de principios, nuestra mirada roza lo urbano y se detiene en la arquitectura construida como visión ciudadana, resultado de una formación académica y responsabilidad profesional del arquitecto, técnico y artista (Cuche, 1999)2. Se disculpará este particular sesgo de una valoración parcial sobre un marco de hechos arquitectónicos y urbanos, que deberían ser contemplados en una investigación integral de futuro. 
En la literatura historiográfica de nuestra disciplina, es posible encontrar distintas acepciones para denominar el tiempo en que se presentan las obras del siglo XX. Últimamente en nuestro medio disciplinar se ha denominado como arquitectura contemporánea costarricense solamente la desarrollada desde 1980 en adelante. Periodización que más allá del intento clarificador, en un tiempo que puede leerse en su acepción como discutible y que admite variantes interpretativas. Una de ellas nos lleva a interrogarnos sobre cuáles serían los puntos de fuerte cesura o notables para determinar en los años ochenta el inicio de esa categorización de la contemporaneidad. O también, cuál sería la ubicación entonces de aquellos arquitectos que realizan obra en este tiempo, habiéndose destacado también en años digamos de una pre-contemporaneidad. Todo parece confirmar, a nuestro criterio, que la arquitectura innovadora de carácter contemporáneo, en una acepción reconocida en la historiografía universal, merece para el caso costarricense un escenario temporal de mayor amplitud. Esto nos lleva a considerar una construcción de la contemporaneidad como una continuidad desde el inicio de la presencia de una arquitectura de imagen y principios fuertemente renovadora hacia el primer tercio del siglo XX, al igual que en el arte costarricense en general.

\section{El marco de una historia de la arquitectura costarricense del siglo $\mathrm{XX}$}

Para una mirada reflexiva de la segunda mitad del siglo XX, es apropiado considerar esta arquitectura de Costa Rica con una referencia a la construcción histórica de su modernidad desde ese primer tercio aludido en el parágrafo anterior. A riesgo de simplificación, podríamos distinguir tiempos o etapas en el acontecer de nuestro arte determinados por la presencia de arquitectos y sus obras, que contribuyen al desarrollo de la cultura arquitectónica del país con las características de su formación y a través de su accionar profesional. También contribuyen hechos culturales referidos a la historia social y política de países determinantes para la cultura arquitectónica de nuestro medio.

Una primera etapa señalada como preámbulo gracias a la presencia de técnicos y profesionales pioneros, que a través de la corriente artística y arquitectónica del Art Decó principalmente, introduce la arquitectura del movimiento moderno de base racional funcionalista. Los hombres más representativos de ese tiempo señalado irán apartándose de la escena en los años sesenta, al igual que los grandes maestros de la arquitectura universal renovadora. Surge así el accionar en una segunda etapa de una pléyade de arquitectos nacionales formados en el exterior. Muchos de ellos actuando hoy profesionalmente. En una tercera etapa ya finisecular, se solapa a la anterior una generación de arquitectos costarricenses que ha procesado su formación disciplinar dentro de fronteras, gracias a la creación de la Escuela de Arquitectura de la Universidad de Costa Rica, enriquecida a su vez con los aportes de profesionales de nuestro continente americano que se integran definitivamente al país ${ }^{3}$.

Recordemos que en la situación de partida hacia nuevos tiempos importa señalar arquitectos que van delineando una nueva cultura arquitectónica en el tiempo que va desde 1930 a 1960 aproximadamente. Esta etapa fue sumamente fecunda en el desarrollo del equipamiento tecnológico de las construcciones, que en lo formal adquiere una renovación estilística al influjo de la cultura europea y norteamericana que llega a estas costas a través de medios documentales, de técnicos extranjeros y de nacionales formados en el exterior. Se destacan tres figuras responsables de los primeros pasos para una nueva arquitectura nacional: José María Barrantes, Francisco Salazar y Paul Ehrenberg, reconocida vanguardia en la construcción de una cultura de

3 Ver la expresada relación entre el destino personal y el trabajo de la arquitectura, emocionado e inédito, en Stagno (1997). El ejercicio de la libertad. México: Ediciones Menhir Libros. $4 \quad$ Ver Fernández (2003). Un país, tres arquitecturas. Cartago: Editorial Tecnológica de Costa Rica. la disciplina arquitectónica. A estos profesionales se debe sumar la actividad de otros técnicos provenientes del extranjero que realizan también obras en este período de vanguardia. Es el caso del arquitecto alemán Ernst Oechsler o del ingeniero formado en Italia, Gastón Bartorelli. El domicilio de este último, en el costado norte del parque de La Sabana, es un ejemplo elocuente de la aceptación de una arquitectura racionalista europea de los años veinte. Esta arquitectura será asumida por aquellos profesionales en sus características constructivas, funcionales y formales, también de la mano primera de la corriente artístico-arquitectónica del Art Decó desde sus vertientes europeas y estadounidenses. Reconociendo su importancia en este período para la cultura costarricense, detengámonos en ella4. 
La arquitectura Decó reconocida con este nombre en la historiografía universal, derivada de la corriente artística del Art Decó consagrada a nivel europeo en la Exposición internacional de Arte Decorativo e Industrial Moderno de París en 1925, adquiere una pronta significación en la arquitectura costarricense. A partir de 1930, en un campo fértil ya preparado al igual que en otros países de América por la reacción antiacadémica a través del Art Nouveau y de expresiones neocoloniales, esta arquitectura abre las puertas en su vertiente especialmente norteamericana al movimiento renovador en la arquitectura a través de primeras manifestaciones que llevan el sello del tiempo presente, expresando con solvencia los nuevos programas arquitectónicos que la dinámica urbana va imponiendo como cines, bancos, hoteles y cafés. En uno de los ejemplos primeros de la presencia de este lenguaje que se aprecia notablemente en las realizaciones arquitectónicas costarricenses, los ingenieros civiles Daniel C. Domínguez, Edwin Góngora y Gastón Bartorelli proyectan el Banco Nacional de Costa Rica en 1944.

Enmarcada en una situación política y social muy particular y al vaivén de los altibajos de las crisis económicas, una arquitectura renovada terminará por imponerse en el país, apoyándose en la actividad del Estado así como en el incipiente empresariado de la industria de la construcción y en el desarrollo inmobiliario en una ciudad que se expande. En un proceso de veinte años, de 1930 a 1950, los demandantes de obra arquitectónica, ya sean públicos o privados, respaldarán una arquitectura entendida como atinada a los tiempos que corren, en el sentido de exponer un lenguaje despojado de estilos del pasado, exhibir mejores calidades constructivas, destacarse por su funcionalidad y expresar nuevos programas arquitectónicos que surgen por el desarrollo económico y social. Como expresión de esto cabe mencionar, por ejemplo, que nuevas tipologías para edificios en altura aparecen en esta etapa. Para el programa habitación: la vivienda por apartamentos. Para el programa de administración y comercio: el edificio particularizado de oficinas, muchas veces combinados con la función comercial como es el caso pionero del Edificio Schifter del arquitecto de origen alemán, Paul Ehrenberg.

En los decenios de los cincuenta y de los sesenta del siglo pasado, asistimos a la paulatina consolidación de la arquitectura contemporánea en Costa Rica. Para entender las transformaciones que se darán en la cultura arquitectónica del país, se deberá tener en cuenta el marco histórico social de la nación. En el decenios de los cuarenta, Costa Rica al igual que el resto de Centroamérica, se encontraba en un nivel de subdesarrollo que recién después de los hechos históricos del 48 comienzan a revertirse más allá de la voluntad política, por transformaciones en la producción y la situación favorable de la economía regional en el intercambio internacional. Costa Rica vivirá a partir de 1950 un período de crecimiento y desarrollo económico interno, que da como resultado niveles de vida más elevados que el resto de la región. Las políticas sociales del bienestar vehiculizaron por parte del Estado la creación de instituciones propicias a la modernización, por lo que el país entero conoció una etapa de desarrollo en todos los órdenes de la vida social (Rojas, 1993). En este sentido, los arquitectos costarricenses asumen un papel en la política de desarrollo, comprometiéndose en la obra pública y llevando a cabo la arquitectura de Estado, que se convierte, a más de sus objetivos programáticos y funcionales, en expresión del progreso nacional. Así como la ideología del desarrollo permea las sociedades latinoamericanas, los arquitectos nacionales, formados en las corrientes de la arquitectura internacional de base funcional racionalista, son ganados por las políticas de gobierno que tienden a

$5 \quad$ El arquitecto chileno Enrique Browne en su obra Una arquitectura en América Latina, describe las características que asume la arquitectura del desarrollo por esos años.

$6 \quad$ En el año 1963, de una nómina publicada en la prensa el 19 de diciembre de 1963 y recopilada por el arquitecto Arnaldo Rohmoser Volio, de 41 integrantes, 17 eran egresados de universidades norteamericanas y 16 lo eran de universidades mexicanas. abrazar institucionalmente variantes de la ideología de desarrollo ${ }^{5}$.

Costa Rica cuenta para ello con una generación de arquitectos graduados en el exterior, la gran mayoría en México y los Estados Unidos de América ${ }^{6}$. Imbuidos de los enfoques de la nueva arquitectura de postguerra, en contacto con profesionales de renombre y realizando una práctica disciplinar, se integran al país y se convierten en actores del desarrollo de la cultura arquitectónica costarricense. Ellos fundaron para la defensa de sus intereses profesionales y académicos la Asociación Costarricense de Arquitectos en 1956, contando en el decenio siguiente con la adhesión de miembros destacados. Entre ellos, es posible detectar autores de obras de relevancia en el plano institucional y la actividad privada. No cuentan todavía con espacios académicos o de intercambio reflexivo y crítico sobre el acontecer de la arquitectura y la ciudad. Estos 
Figura 1. Banco de Costa Rica, San José. Fotografía por Carlos Altezor.
$7 \quad$ La Asociación Costarricense de Arquitectos (ACA) fue fundada en 1956. Sus miembros fundadores fueron los arquitectos: Crespo Perera, Dobles Rodríguez, Dominguez Parraga, Ehremberg Brinkman, Escalante Van Patten, Garrido Llovera, Gordienko Orlich, Maroto Montejo, Masis Dibiasi, Padilla Quesada, Quirós Alvarado, Salazar Quesada, Sotela Pacheco y Vargas Vargas.

$8 \quad$ En los Estados Unidos de América, a partir de la década de los cincuenta, se concreta una tipología arquitectónica que se ha reconocido como resultado del desarrollo tecnológico de nuevos materiales y procedimientos constructivos, de una tradición de edificación en altura desde fines del siglo XIX y de la potencialidad financiera del capitalismo norteamericano. Así, cuando en 1952 se inaugura el edificio Lever en Nueva York, una nueva era se abre para el programa de edificios administrativos que influirán en todo el mundo. Organizados formalmente en dos paralelepípedos uno horizontal de una o dos plantas y otro vertical de varios pisos, hacen alardes del uso de la tecnología en acero, hormigón armado, vidrio y acondicionamientos artificiales, sin referencias historicistas y estrictamente funcionales. Su imagen urbana más notable es la producida por el cerramiento de fachadas en grandes extensiones vidriadas -curtain wall- reflejantes del entorno. abrían de esperar el establecimiento de la Escuela de Arquitectura de la Universidad de Costa Rica en los años setenta y el accionar dinámico del Colegio de Arquitectos para participar de una mayor actividad en la cultura nacional ${ }^{7}$.

En este período, la característica más saliente que presenta la arquitectura es una configuración inspirada en modelos internacionales y preferentemente tipologías norteamericanas y europeas, que como ya dijimos, se manifiesta elocuentemente en la obra de Estado. La arquitectura institucional mayor adopta la tipología de la construcción en altura ${ }^{8}$. Sin relación con el contexto urbano donde se inserta, emerge en el tejido de la ciudad de manera aislada, cual filamentos de concreto sobre el terreno, exigiendo un espacio propio y generoso para accesos y estacionamientos. En un primer momento, durante la década de los sesenta, estos edificios se ubican en los ejes urbanos de relevancia en la ciudad de San José. Es el caso por ejemplo de la Caja Costarricense del Seguro Social, de los arquitectos Carlos Vinocour y Rafael Sotela, sobre la Avenida 2, o el Banco de Costa Rica, obra del arquitecto Hernán Arguedas. También esa tipología en altura emerge en áreas laterales, dinamizando espacios urbanos y centrales generando así una actividad funcional en ámbitos paisajístico de relevancia, como es el caso del edificio para el Instituto Nacional de Seguros, obra de los arquitectos Roberto Villalobos y Rafael Esquivel, sobre el costado norte de la Plaza España, o de la obra para el Instituto Costarricense de Electricidad (ICE) en el lado norte del parque de La Sabana del arquitecto Rafael Sotela.

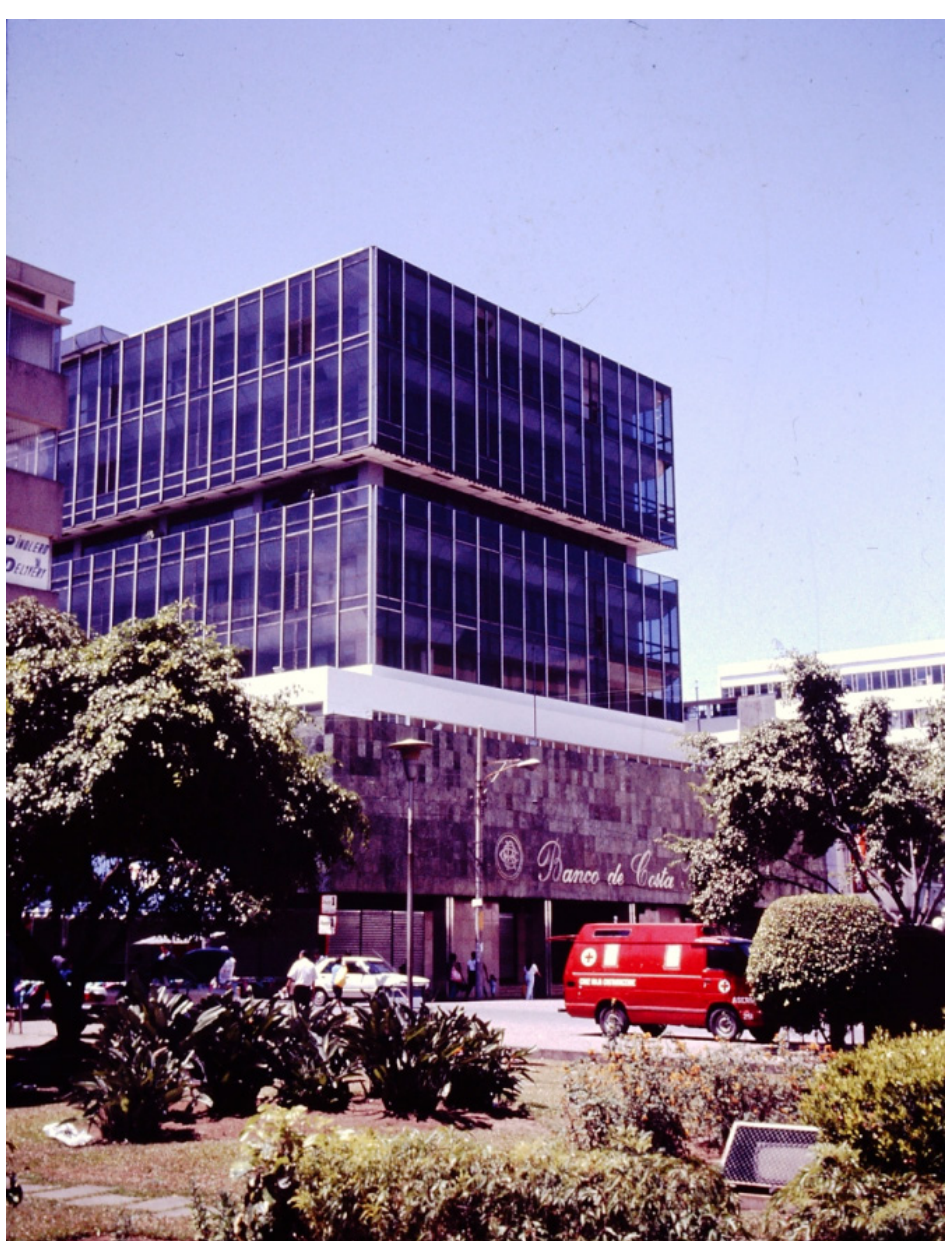

Estas tipologías aportan características distinguibles a la trama de la ciudad por su capacidad de perfilar, como se ha dicho, las calles céntricas sin descuidar las protecciones para un especial clima tropical con adecuados porticados para proteger del sol y de la lluvia, por sus modestas alturas en general o por la atenta referencia al paisaje urbano inmediato. Esta arquitectura hace gala de un expresionismo estructural afín a una corriente arquitectónica que se expresa además en América Latina, exhibiendo el uso predominante del concreto armado, posteriormente los avances técnicos en carpintería metálica y el uso del vidriado extenso en las fachadas. Esto último será una de las características más distinguibles en las fachadas de los edificios y que continuará con desmedido uso en los decenios siguientes. También se lleva adelante el programa de integración del arte pictórico y escultórico a los edificios, con antecedentes en la integración de las artes propio de las vanguardias europeas y el desarrollo del muralismo mexicano. Para el caso de Costa Rica, ello será respaldado por ley y con ejemplos importantes de obras que irán incorporando a los edificios, la escultura interna y externa, así como la pintura mural, artes todas debidas a artistas nacionales. Por citar un ejemplo, Manuel de La Cruz González realiza en la sede del Banco Anglo Costarricense, avenida 2 y sobre la calle 3, un mural en el espacio exterior y de acceso al banco. 
$9 \quad$ Este punto merece ser profundizado en sus detalles a través de una investigación particularizada, aprovechando la vigente actividad de muchos de los protagonistas de la construcción académica de la Escuela. Además, es interesante apreciar que su establecimiento despertará la creación de instituciones privadas para la enseñanza de la disciplina en el país, generando con aquellas conexiones eficaces a la par de formar profesionales de valor.

\section{Ver la situación general sobre la cultura} costarricense en Cuevas, R. (1996). El punto sobre la I. Políticas culturales en Costa Rica (19481990). San José: Ministerio de Cultura, Juventud y Deportes.

$11 \quad$ Nos referimos particularmente a los arquitectos Jorge Bertheau, Franz Beer, Rafael Angel García y Edgar Brenes.

12 La primera graduación estuvo constituida por los arquitectos Ofelia Sanou, Gloria Wang, Ibo Bonilla, Alvaro Cambefort, Stephen Chaverri, Carlos Gutierrez, Carlos Jiménez, Manuel Morales y Guillermo Navarro.
En estos años se concretan obras por parte del Estado que trascienden su ejecución única y aislada en el territorio urbano, para procesarse en un conjunto de edificios de características específicas. Esta manera de sumarle "partes" funcionales a programas determinados en un solo acto proyectual al territorio, urbano o suburbano, constituye una herencia del movimiento de la arquitectura internacional y del urbanismo, desarrollado a partir de las guerras mundiales, privilegiando la construcción de un "zonning" en las ciudades aprobados por la nueva ciencia del urbanismo. En nuestro medio, dos ejemplos son los más elocuentes. El primero de ellos tiene que ver con el establecimiento de la Ciudad Universitaria Rodrigo Facio en San Pedro de Montes de Oca. Esta constituye con el tiempo la mejor muestra del desarrollo de la cultura arquitectónica contemporánea costarricense. Exhibiendo desde edificios iniciales muy afines a la ortodoxia del movimiento racionalista, hasta obras actuales de buena calidad constructiva, funcional y plástica, que hacen de este conjunto, no suficientemente valorado, el mejor de América Central. El segundo ejemplo se realiza en terrenos netamente urbanos conocidos actualmente como La Corte, en sitio que dejara la Universidad de Costa Rica en oportunidad de su traslado a una nueva sede en San Pedro de Montes de Oca. Ese conjunto nuclea las actividades centrales del Poder Judicial nacional y sus dependencias, mostrando una gran unidad de diseño y calidad arquitectónica que realzan el sentido emblemático de uno de los poderes constitucionales del Estado.

Aproximándonos a una visión de la arquitectura costarricense de los tres últimos decenios del siglo XX, observamos que ciertos temas vertebran la situación de la cultura en el país, construyendo su identidad en el marco latinoamericano. Así al inicio de la década de los setenta, algunas manifestaciones comienzan a expresarse como resultado de hechos que se perfilaron en décadas anteriores de continuidades en la consolidación de una arquitectura de nuestro tiempo. En esta década, se concreta la enseñanza de la disciplina arquitectónica por primera vez en el país, fundándose la Escuela de Arquitectura de la Universidad de Costa Rica. El Colegio de Arquitectos revitaliza su accionar y continúan con la integración al país de nacionales y extranjeros graduados en el exterior. A su vez, surgen centros de enseñanza de arquitectura vinculados a universidades privadas y se consolida la vía del concurso para asignación de proyectos y obras para el sector público y privado. Estos acontecimientos van jalonando para los años siguientes un tiempo cualitativamente distinto y de calidades para la cultura de la disciplina. Resta entonces describir e interpretar en ese marco algunos tópicos principales de la arquitectura nacional y sus distintas manifestaciones.

\section{La enseñanza de la arquitectura en la Universidad de Costa Rica}

El hecho más relevante del siglo XX, el punto de inflexión en el escenario de la cultura arquitectónica es la creación, en el decenio de los setenta, de la Escuela de Arquitectura de la Universidad de Costa Rica9 ${ }^{9}$. Para ese aserto, es necesario recordar la situación social y cultural del país ${ }^{10}$. El medio académico y profesional se encontraba maduro para el establecimiento de los estudios nacionales de arquitectura. El país contaba hacia esos años con un excelente plantel de profesionales graduados en el exterior, con conocimiento de un oficio, de un diseño que era compartido universalmente y con experiencia de obra en el ámbito costarricense. A toda justicia, es la iniciativa de un pequeño grupo de impulsores que se pone al hombro la tarea ${ }^{11}$ de iniciar los estudios desde una experiencia de posgrado en el exterior y en los revulsivos años sesenta de la cultura europea que evitan aplicar mecánica y acríticamente modelos didácticos agotados. Estos pondrán en marcha aquellos estudios y la historia de los primeros años de la Escuela de Arquitectura se desarrolla en un marco de polémicas que trasciende muchas veces su ámbito para consolidarse posteriormente, no sin experimentar variadas crisis. La primera graduación de arquitectos es de $1977^{12}$, producto de un enfoque innovador en la metodología de enseñanza aprendizaje, crítica, reflexiva y cuestionadora, en una actitud de pensar con la propia cabeza y enmarcada en la realidad nacional sobre la cual se pretende operar desde la disciplina. Las actividades docentes de la Escuela de Arquitectura en el lapso transcurrido hasta hoy ameritan desde nuestro punto de vista una mirada heurística de profundidad ante un episodio de 
innegable presencia cultural en el país. Acercándonos a conmemorar dentro de poco el medio siglo de existencia de la Escuela de Arquitectura, la oportunidad parece propicia para balances y transformaciones programáticas académicas: ¿Incorporar Diseño, Urbanismo, Institutos de investigación de apoyo a la enseñanza? El desafío del futuro lo dirá...

Como característica de este tiempo, es posible señalar que aquellos arquitectos que inicialmente realizan obras después de la segunda mitad del siglo mantienen una fuerte presencia en la cultura artística y arquitectónica del país mediante una continuidad de trabajo, aunque cediendo espacios paulatinamente a una generación de ya graduados en el medio y profesionales provenientes del exterior. Unos y otros acrecientan un reconocimiento avalado por la obtención de premios en concursos públicos realizados, destaques en encuentros académicos, así como la selección de ejemplos de sus obras en publicaciones, tanto bibliográficas como en revistas especializadas del espacio nacional y latinoamericano.

\section{Identidad de la arquitectura costarricense}

Estas continuidades hacen a la construcción de la identidad de una arquitectura de lo local frente a la globalidad, de tiempo y lugar, temas siempre presentes y acuciantes para los latinoamericanos como consecuencia del proceso de dominación cultural de los países desarrollados del hemisferio norte a lo largo de la historia. Esta situación sigue hoy vigente en momentos en que la humanidad experimenta un gran cambio hacia el surgimiento del llamado mundo globalizado y, correlativamente, los primeros pasos de un colosal cambio cultural. Estas actitudes de identidad que nos remiten a posiciones antiacadémicas de raigambre hispanoamericana, por ejemplo en el llamado estilo neocolonial, expresadas en la arquitectura costarricense desde los años veinte a los años cuarenta principalmente. Dicha arquitectura que se entendía propia de una tradición constructiva y formal que tenía en cuenta el paisaje natural, el ambiente y atinada a los modos de vida de los hombres. Una línea de pensamiento en esos temas se hace presente en el desarrollo de la arquitectura costarricense a través del discurso y obra de connotados arquitectos de la segunda mitad del siglo. A veces manifestada con elocuencia o solo parcialmente con el correr del tiempo. La literatura que estos mismos arquitectos elaboran en pos de una obra que caracteriza la identidad es también elocuente en estos últimos años, pero como nos advierte el arquitecto Jorge Grané en "La mexicanización de nuestra arquitectura", publicada en la revista IMAGO de julio de 1998, se nos presenta a una tradicional arquitectura de México como ideal para una obra de "identidad" costarricense en desmedro de "una arquitectura propia con matices locales y con la satisfacción de crear un producto que ha salido de nuestras fuentes" (Grané, 1998, p.43).

Aquella actitud parece contradecir con la formación recibida por la gran mayoría de ellos en México, los Estados Unidos o en otros ámbitos americanos, teñida de la ideología de la arquitectura racionalista y la influencia de los grandes maestros del llamado también Estilo Internacional. Creemos que aquella línea de pensamiento y acción se manifiesta en forma permanente y como sustrato, por lo que debe ser valorada como uno de los rasgos más señalados de una identidad de la cultura arquitectónica costarricense. Esta línea se caracteriza, por ejemplo, en la obra del arquitecto Hernán Jiménez y su reconocimiento de la influencia, en su formación en la Escuela Nacional de Arquitectura de la UNAM en México de los sesenta, de los grandes maestros de la arquitectura racionalista europea. A su regreso a Costa Rica, realiza una obra de cuidado formal y esmerada técnica. Obtiene en 1978 el primer premio del concurso para la sede del Colegio Federado de Ingenieros y Arquitectos, obra emblemática de la arquitectura contemporánea costarricense para toda una generación de profesionales. Desde la enseñanza en el taller de diseño en la Escuela de Arquitectura de la Universidad de Costa Rica, desarrolla una línea de valoración de aspectos que hacen a la identidad nacional del arte y la arquitectura y un reconocimiento en ésta de una cultura vernácula. Su vivienda propia en Santa Cruz de Turrialba de 1994, es asumida personalmente como un compendio de su pensamiento hacia una arquitectura vernácula por su escala, por su integración al paisaje, vinculación espacial interior- exterior y el uso de 
materiales del lugar.

Otro ejemplo de actividad profesional comprometida en la búsqueda de una obra de identidad nacional, es el caso del arquitecto Bruno Stagno proveniente de otro país americano. Este arquitecto se gradúa en la Facultad de Arquitectura de la Universidad Católica de Chile, realiza estudios de posgrado en Francia y en la tradición del maestro Le Corbusier se nutre de las teorías del racionalismo imperante en la arquitectura. De esta manera, habiendo recibido un bagaje intelectual y artístico-arquitectónico europeo, ingresa al país que lo acoge y comienza a elaborar un pensamiento original, acorde con aquellas permanencias de una tradición cultural enraizada en el medio, un territorio, un paisaje y un clima que no determinan su arquitectura, pero sí la condicionan. Sin afiliaciones a modas ni clichés, el diseño y obra resultantes demuestran que se puede innovar desde nuestras realidades con posturas propias.
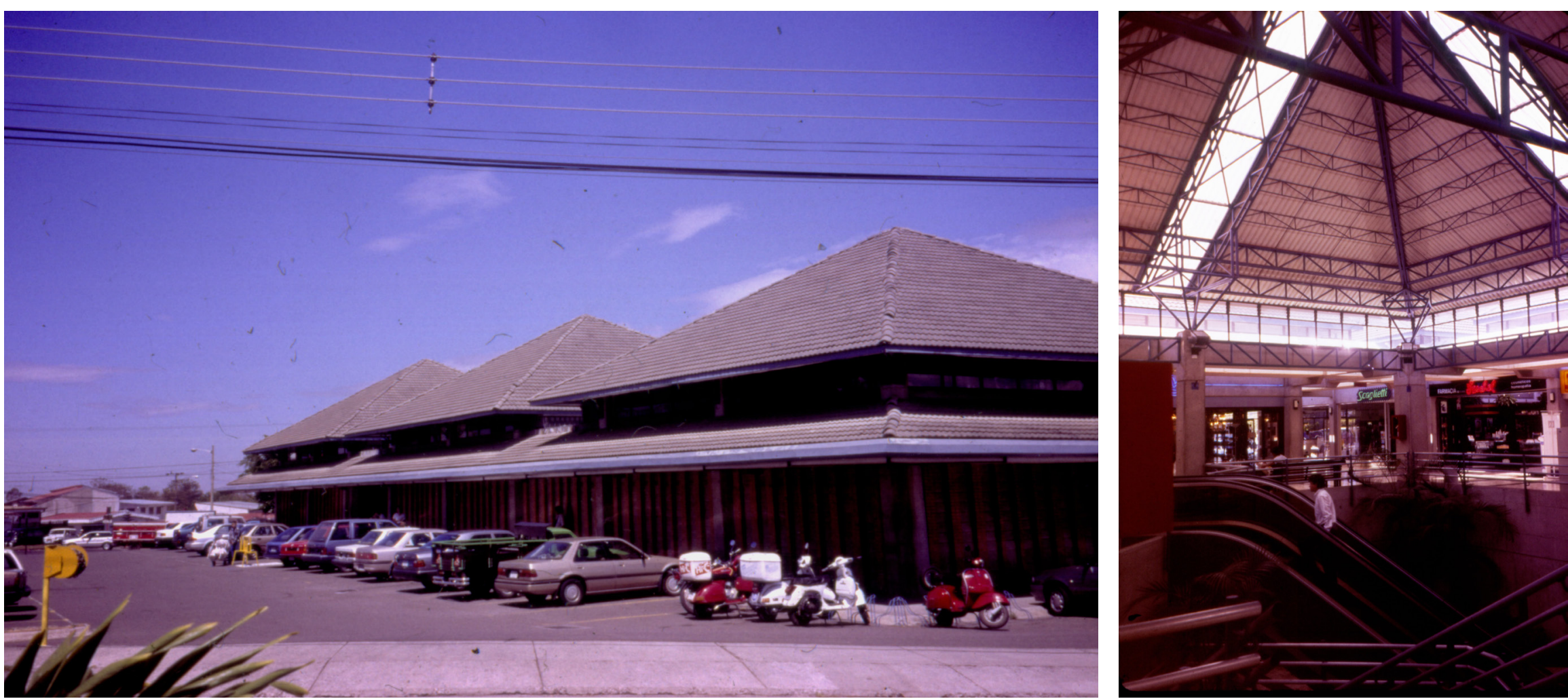

Figuras 2 y 3 . Centro Comercial Plaza Mayor Rohrmoser. Exterior e Interior. Fotografias por Carlos Altezor.
Figura 4. Contraloría General, San José. Fotografía por Carlos Altezor.
Anotamos otra característica de fin de siglo: la permanencia de la influencia de vertientes doctrinarias así como posturas acríticas con respecto a la arquitectura, pero también sobre distintas manifestaciones artísticas provenientes de los grandes centros de irradiación del discurso y la práctica del diseño, fundamentalmente desde los Estados Unidos de Norte América y el espacio europeo. Más allá de inevitables y sanas influencias en la formalización de los modelos de diseño en el arte y la arquitectura, es apreciable en nuestro medio la incorporación irreflexiva y descontextualizada de una arquitectura de revistas, ejemplos de las obras de prestigiosas firmas y oficinas internacionales. Arquitecturas de buen nivel tecnológico, pero que no guardan armonía con el entorno urbano o paisajístico, pues son soluciones asumidas que más allá de una buena resolución funcional o de brindarnos una imagen de avanzada o en consonancia con las demandas internacionales, no parecen resolver una adecuada solución económica con respecto al clima. Así se ejecuta muchas veces una arquitectura que confunde a la vez que degrada el medio ambiente. Al igual que aquellas obras que detrás de últimas novedades al ser incorporadas irreflexivamente al medio donde se emplazan merecen ser cuestionadas al acusar una desmedida relación entre la identidad cultural y la presencia de modelos descontextualizados.

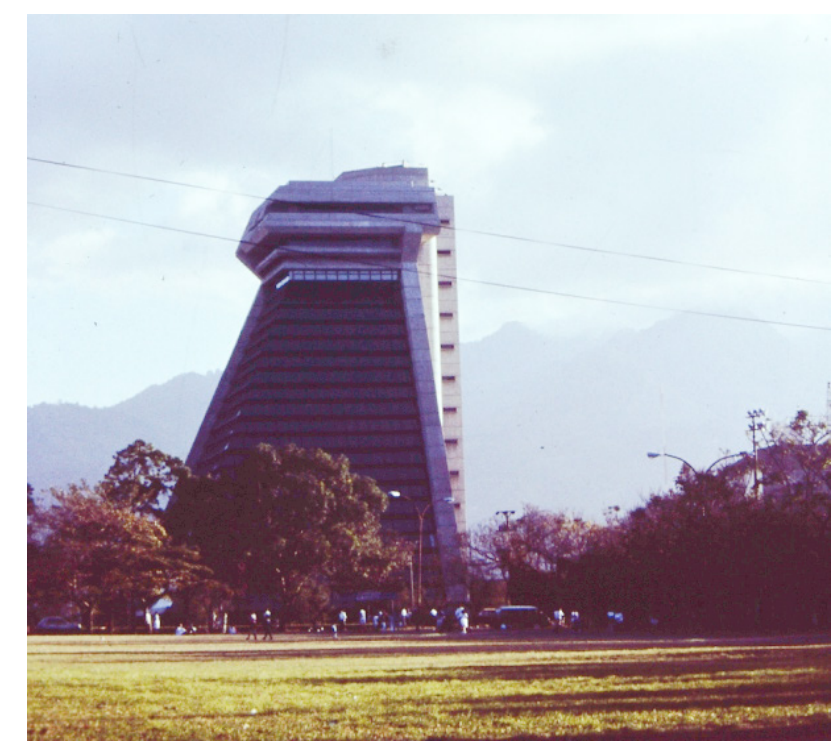


Como alternativa a lo anterior, asistimos en el presente a una corriente crítica que plantea una arquitectura en acuerdo a la realidad económico-social nacional. Esta corriente revitaliza y pone en debate posturas que estuvieron de alguna manera siempre presentes en la cultura de la disciplina desde mediados del siglo como ya hemos señalado, especialmente en las cátedras universitarias. Hemos denominado a esta postura como la de los tres respetos a ciertos principios: el respeto a la identidad de una arquitectura con raíces en el pasado, relacionada con la tradición constructiva que no niega referentes internacionales; el respeto a un entorno natural y paisajístico que en el país adquiere altos valores reconocidos internacionalmente como una arquitectura que no agrede al medio natural y se integra a él con armonía; el respeto por último, al paisaje urbano con obras que hacen al mejoramiento de la calidad de vida de los ciudadanos. Una obra como ejemplo para el caso de un programa particular: el centro comercial de San Pedro, obra del arquitecto Jorge Bertheau y asociados. Un nuevo programa para la atención ciudadana, logrado con una integración respetuosa con el ambiente urbano y su uso social. Desde estos puntos de vista, obras calificadas han sido construidas últimamente en distintos programas por reconocidos arquitectos nacionales que fundamentan su arte en un meditado discurso, acompañado en algún caso por la actividad de centros de investigación y difusión a los que hay que prestar particular atención. El mismo se ha desarrollado pacientemente, enriquecido en la labor docente y la divulgación, y ha recibido por sus planteos de una arquitectura de alternativa importantes reconocimientos a nivel nacional e internacional. Entre los autores de ese discurso y con obras que son representativas de esas nuevas actitudes valoradas en las Bienales de Arquitectura y Urbanismo de Costa Rica, se hallan los arquitectos Víctor Cañas, Hernán Jiménez, Alberto Liner, A. Quesada, Jaime Rouillón, Bruno Stagno, Edwin Villalta, Julia Van Wilpe, entre otros.

Un sesgo interesante es la pregunta si la arquitectura de Costa Rica puede ser considerada producto-frontera entre dos vertientes en el siglo XX entre las arquitecturas del hemisferio norte americano, especialmente mexicanas, y arquitecturas de la cultura sudamericana, a través de arquitectos nacionales y extranjeros que se asientan en el país. Por otro lado, si también por el conocimiento teórico e historiográfico presentes en bibliografías, documentos y comunicaciones académicas de seminarios, encuentros y congresos. Sin duda que sí... No olvidemos que desde inicios del siglo XX, se visualiza como una invariante o una constante que se desarrolla en el correr de esa centuria. En las figuras paradigmáticas de un costarricense que se forma a sí mismo al margen de las academias, como Barrantes, de un josefino que se gradúa en América del Norte, al igual que Salazar, o de un extranjero, Ehrenberg, en este caso proveniente del ámbito europeo y que se radica definitivamente en Costa Rica. Esta será la tónica durante el siglo: el destaque de la creatividad de los arquitectos nacionales formados dentro y

13 En una profundización heurística de esta temática hay que recordar el peso que adquiere la llamada "literatura gris" vinculada al hacer administrativo, de actividad académica y de apoyo a la didáctica.

14 A la fecha de realización de este trabajo, las Bienales de Arquitectura y Urbanismo de Costa Rica han sido cinco. La última fue realizada en 1996 bajo la denominación "Tierra, tiempo y espíritu en la Arquitectura". Vale recuperar ponencias de los arquitectos costarricenses para su análisis y valoración.

15 La revista Habitar, órgano del Colegio de Arquitectos, vio la luz en 1976 y se continuó editando hasta 1995. En estos diecinueve años se han publicado cuarenta números, conteniendo diversos temas.

16 Ver colección de artículos periodísticos diario La Nación y Revista Habitar: "Aventuras de Félix, el Arquitecto". fuera del país y los aportes de profesionales sudamericanos y europeos, integrados plenamente a la cultura de la arquitectura costarricense.

\section{Literatura para el conocimiento y el debate disciplinar}

Una característica de la mayor importancia en la segunda mitad del siglo es sin duda el desarrollo de la especulación teórica e historiográfica sobre la arquitectura y sus variadas expresiones de comunicación por parte de los arquitectos nacionales. En los últimos decenios, se aprecia una mejor disposición a la interna del medio profesional para el debate sobre la arquitectura y la ciudad y se determinan vías para la información y la reflexión. Los arquitectos costarricenses publican y difunden su pensamiento más allá de ocasionales artículos de prensa o de aquella literatura circunscripta al campo de la enseñanza ${ }^{13}$. Los canales abiertos de relación con el medio latinoamericano determinan el conocimiento y los aportes de ideas y experiencias interdisciplinares. Se establece en Costa Rica Bienales de Arquitectura y Urbanismo ${ }^{14}$, en donde se publica más sobre arquitectura en los medios de prensa escrita y hasta las ondas de radio y televisión prestan su concurso para la difusión de temas, ante todo sobre la problemática urbana. De esta manera, la obra arquitectónica costarricense comienza a ser reconocida no sólo en el país sino también fuera de fronteras. Particular mérito y destaque merece la edición regular de la revista Habitar del Colegio de Arquitectos, vehículo idóneo para el conocimiento y difusión de nuestra cultura y medio de contacto 
entre profesionales que abre caminos de reflexión a través de sus contenidos, despertando a su vez iniciativas para otras publicaciones hacia fines del siglo ${ }^{15}$. Debe registrarse la interesante comunicación con el público a través de secciones de prensa como son los artículos del arquitecto Jorge Grané en referencia a obras y situaciones urbanas. Además de sus artículos en la revista Habitar como Félix el Arquitecto, en la mejor tradición de un humor crítico hispanoamericano, a la par de construir entre líneas para los más avisados una constelación de relaciones inter pares y con la sociedad ${ }^{16}$.

En los últimos decenios del siglo se irán conociendo obras de otros investigadores del país, particularmente arquitectos. Estos trabajos de real valía serán bienvenidos, ya que abrieron nuevas oportunidades de reflexión sobre el arte de la arquitectura que nos estaba dejando el siglo XX en Costa Rica. En buena hora estos autores se manifestaron aportando diversas miradas que se hacían necesarias. Algunos con un enfoque netamente de análisis histórico, en el entendido de registrar preferentemente obras y hechos del acontecer que van jalonando la segunda mitad del siglo. Otros, apostando a definir el tiempo de la arquitectura contemporánea en Costa Rica con aportes metodológicos de relevancia y destacando un marco teórico donde se ubican autores de realizaciones que se juzgan ejemplares de nuestro tiempo.

Especial mención debe hacerse en la cultura arquitectónica del fin de siglo costarricense a la obra historiográfica que llevan adelante en estos años diversos arquitectos, que desde la docencia o fuera de ella, publican textos imprescindibles que la disciplina siempre se debe. Tales son los casos de los arquitectos Ofelia Sanou, lleana Vives, Luis Diego Barahona, Franz Beer, Juan Bernal Ponce, Jorge Grané, Raúl Hernández, Bruno Stagno, Roberto Villalobos, entre otros. El ejemplo más elocuente lo constituye la publicación de la Historia de la Arquitectura en Costa Rica, editada por el arquitecto Enrique Garnier e historiadora Elizabeth Fonseca en el año 1998. Obra de imprescindible consulta por la calidad de sus contenidos, por su enfoque totalizador de la historia de la cultura arquitectónica y urbanística y la idoneidad de los autores intervinientes en la temática tratada. Es un verdadero hito en la historiografía de nuestra disciplina ya que es el resultado además de un trabajo en equipo no sólo integrado por arquitectos de una renovada generación sino también por investigadores de otras disciplinas (Fonseca \& Garnier, 1998). Esta obra no desmerece otras donde se encara un desarrollo de una arquitectura actualizada a los nuevos tiempos de la contemporaneidad (Barahona, 2000).

\section{Nuevos programas para la arquitectura}

Una característica notable de estos decenios de fin de siglo es la referente al programa de habitación individual y colectiva, y vaya si hay un programa y una actuación más apreciada por los arquitectos que el tema de la vivienda, propia o ajena, individual 0 colectiva. Este programa, por el mayor número de oportunidades que otorga, es el que permite acercarnos a la obra de un arquitecto o de una generación de profesionales. Comentadas en diversas publicaciones y especialmente en la revista Habitar, visitadas por colegas y exhibidas como ejemplo del hacer de cada profesional constituyen una apertura sin prejuicios a la consideración académica y profesional que años atrás no tenía lugar. En general podemos leer en estas obras distintas tendencias de las cuales se hacen eco sus autores y que críticos y observadores atentos interpretan y clasifican a su entender (Barahona, 2000). Desde soluciones atentas a referencias vernáculas como es el caso de la vivienda para el arquitecto Franz Beer, proyectada por Jorge Bertheau en Curridabat, como expresiones respetuosas de la intimidad y del sentido de refugio propio de los costarricenses, como es el caso de la vivienda propia también ejecutada por el arquitecto Víctor Cañas en la misma zona de urbanización. 


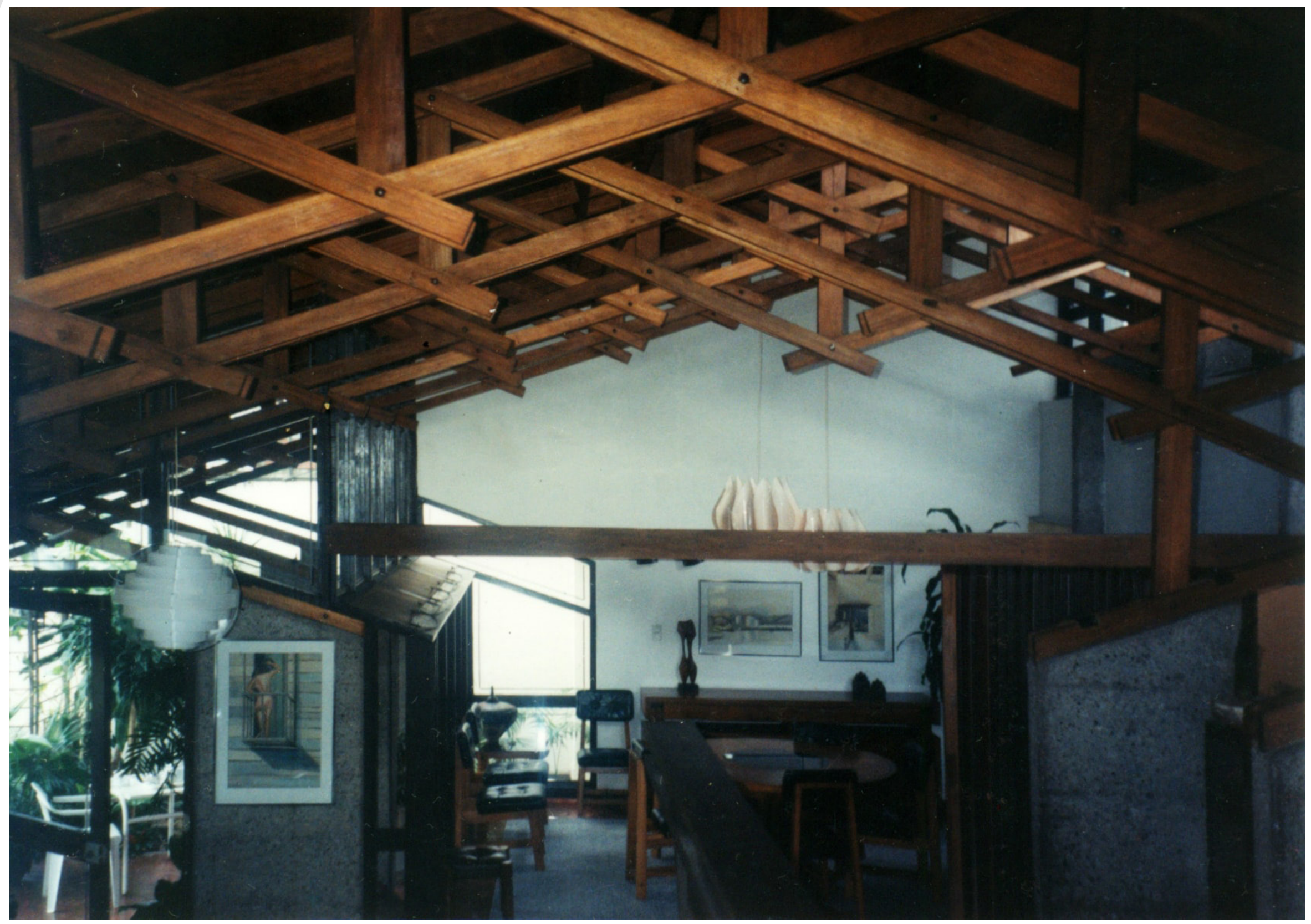

Figura 5. Vivienda Franz Beer. Fotografía por Carlos Altezor.
En el programa de viviendas colectivas, debemos destacar el notable desarrollo que en las últimas dos décadas del siglo adquiere los conjuntos habitacionales en altura, ubicación en las zonas residenciales preferidas por las clases altas josefinas. Estos edificios en altura, al igual que los destinados a oficinas, constituyen expresión de una alta tecnología ingenieril que se expande notablemente en el país de la segunda mitad del siglo en adelante. Vinculado a esta temática, se señala la notable difusión del programa hotelero, acompasando el desarrollo turístico en sus dos grandes versiones: hotelería de ciudad y de playa. En esta última vinculada a la función vacacional, se aprecia una esmerada atención al medio ambiente y las consideraciones paisajísticas naturales de costa y de montaña propias de Costa Rica. En el medio urbano, en San José particularmente, es paradigma de la nueva hotelería el hotel Europa Zurquí, obra del arquitecto Rolando Barahona. Como alojamiento turístico de costa, el proyecto de arquitecto Hernan Ortiz, en el marco del Sistema Nacional de Albergues, es un diseño respetuoso del ambiente costero y de la arquitectura vernácula en correcta relación con el paisaje natural, el cual será un referente obligado a tener en consideración. En una relación de vivienda permanente y entorno, es destacable el partido asumido por la arquitecta Julia Van Wilpe para sus proyectos de urbanizaciones de costa marítima en el Pacífico y especialmente el conjunto residencial de montaña en Santa Ana.

También en estos últimos años, los arquitectos costarricenses han intervenido en programas urbanos, particularmente en lo que tiene que ver con las áreas verdes inmersas en la ciudad, especialmente San José. Es un meritorio esfuerzo que se destaca en el entorno latinoamericano, problemáticamente difícil por las presiones del mercado inmobiliario que se resiste a entregar áreas para el uso ciudadano y el mejoramiento ambiental de nuestras ciudades. En la ciudad de San José, soluciones de equipamiento para espacios públicos como peatonalizaciones, parques y plazas, han posibilitado la recuperación de sitios para el uso ciudadano que nuestras ciudades, hoy más que nunca, necesitan apropiarse y que por derecho les corresponde. Los concursos y los encargos de ejecución han abierto en nuestro medio un camino de experiencias y el acondicionamiento de áreas de potencial uso turístico y ciudadano han contado con la capacidad creativa de los profesionales. Tanto sea en programas puntuales, como 
en la formalización estudiada y medida de una arquitectura para el uso respetuoso del entorno. Sin duda, el programa de la contemporaneidad en este campo la constituye la plaza urbana y en ella, como obra mayor, el complejo de la Plaza de la Cultura, creación de los arquitectos Jorge Bertheau, Edgar Vargas y Jorge Borbón. Además, dos espacios centrales de la ciudad capital se han recuperado para lugares públicos: la Plaza de la Democracia y la Plaza de las Garantías Sociales, ambas proyecto del arquitecto Edwin Villalta. Obras concebidas de acuerdo con concepciones actuales sobre el paisaje urbano, donde las nuevas áreas verdes no constituyen solamente plazas homenajes ni están al servicio de arquitecturas notables, permitiendo el uso y el pleno recorrido del paseante, a más de la apertura de posibilidades de uso en el subsuelo para programas diversos. Un nuevo parque al sur de la ciudad se agrega para el nuevo siglo: el Parque de la Paz, que, sumado al desarrollo del Parque de La Sabana en una realización desde la década de los años setenta, prestigia al arquitecto A. Quesada. Políticas de ejecución de plazas, parques urbanos y nacionales no son manifestaciones frecuentes en nuestra América, pues vinculadas al tema de intereses diversos sobre el territorio y el suelo urbanizable, no son fáciles de imponer en aras del bien común del ciudadano.

\section{El patrimonio arquitectónico a fines del siglo}

En estas temáticas de fin de siglo, apreciamos el desarrollo de una línea de pensamiento y de práctica sobre el patrimonio arquitectónico y urbano nacional en sus posibilidades de conservación y uso. Este apoyándose en la fuerza de la ley y en el organismo institucional correspondiente: el Departamento de Patrimonio Nacional del Ministerio de Cultura, Juventud y Deportes ${ }^{17}$, respaldado también por un emprendimiento como el ICOMOS de Costa Rica ${ }^{18}$. El patrimonio se encuentra hoy protegido, pero no exento de peligros producto de la ignorancia, de intereses de distinto tipo o de decisiones individuales bien intencionadas pero equivocadas. Actualmente, la línea de re-funcionalización de edificios de tiempos pasados permite recuperar para la memoria y para el uso actual ejemplos de arquitectura y entornos urbanos valiosos ${ }^{19}$. Valga también como ejemplo en este período la puesta en valor de un edificio otrora destinado a la producción industrial, la Antigua Fábrica Nacional de Licores, ubicada en el costado este de la Plaza España en San José. Una feliz y oportuna decisión estatal logró recuperar la obra a través de un llamado a concurso público para un Centro de Nacional de la Cultura (CENAC) el año 1991. Ganadores de dicho concurso fueron los arquitectos Hernán Jiménez, Nicolás Sánchez, Marcos Valverde y Nuria Gutiérrez distinguidos en la II Bienal de Arquitectura y Urbanismo de Costa Rica con el Premio Nacional de Arquitectura. Se demuestra así que la atención a una política de conservación y puesta en valor de edificios de interés patrimonial permite salvar para la cultura del país, bienes que no merecieron atención en épocas anteriores. Además se debe tomar en cuenta las dos últimas bienales de fin de siglo realizadas en Costa Rica, apreciando distinciones en la II Bienal del año 1994 a los arquitectos Hernán Jiménez, Nicolás Sánchez y Marcos Valverde (Premio en la categoría Restauración y Conservación Arquitectónica por la obra Centro Nacional de la Cultura); arquitectos Eric Chávez y William Monge (Premio en la categoría Restauración y Conservación Arquitectónica, Mención honorífica, por la restauración del Teatro Nacional); arquitecto Alberto Linner (Mención Honorífica en la categoría Diseño Arquitectónico por su obra Hospital Max Teherán Paez); arquitecto Bruno Stagno (Mención honorífica por su obra, Edificio Dinca); arquitecta Julia Van Wilpe (Mención Honorífica por su proyecto de Casa Kors en Santa Ana); arquitecto Juan Bernal Ponce (Premio en la categoría Teoría, Historia y Crítica por el libro Ciudades del Caribe y Centroamérica); arquitectos Jaime Rouillón y Edwin Villalta en la categoría proyectos no construidos por sus trabajos en Casa Straight y Vivienda Ecológica en Carrillos de Poas. Para la III Bienal del año 1996 se destacan el arquitecto Rolando Barahona (Premio Categoría Diseño Arquitectónico por el Hotel Europa Zurqui ); el arquitecto Bruno Stagno (Premio Mejor Proyecto de Arquitectura por su Salón de Exposiciones de Vehículos Ford); arquitecto Jaime Rouillón (Gran Premio por ampliación de Hotel y Mención Honorífica por su obra para Estudios Conte); arquitectos J. Garro y Percy Zamora (Mención Honorífica en Restauración Arquitectónica por la reconversión de la Penitenciaría Central en Museo del Niño); arquitecto William Monge (Mención Honorífica en Diseño Urbano por la Terminal para Trasporte Interurbano y también en la 
20 En esta obra Huyssen, que ha cultivado la temática del tiempo y la memoria, la identidad nacional, la vanguardia, el modernismo y las ciudades en la cultura y el arte desde 1960, nos dice que, en la década de 1990, en el mundo entero se asistió a una explosión sin precedentes de la cultura de la memoria. Inmerso en un mundo de cambios para bien y para mal, éstos no generaron una nueva visión del futuro. Da la sensación de que, en la actualidad, el pasado es evocado para proveer aquello que no logró brindar el futuro en los imaginarios previos del siglo XX.
Categoría Restauración Arquitectónica por su obra referida a la calle frontal de la Plaza del Teatro Nacional). De todas maneras, parece prudente mantener una alerta sobre la línea de protección patrimonial de la arquitectura que no contemple cierta integralidad con las disciplinas de las ciencias sociales y tecnológicas (Hyussen, 2001) ${ }^{20}$.

\section{Conclusiones}

En páginas anteriores pretendimos dar nuestra particular versión de algunos aspectos que creemos notables de la cultura arquitectónica costarricense a fines del siglo. Un cultura particularizada en un panorama de políticas culturales del país. Esos aspectos han signado el tiempo pasado y manifiestan a nuestro parecer su vitalidad en el nuevo siglo. Cuatro temas llamaron nuestra atención: enseñanza académica, formación del arquitecto, identidad de la arquitectura costarricense en el concierto regional, desarrollo de la actividad especulativa en las áreas de teoría e historia de la disciplina y el patrimonio arquitectónico y urbano de la nación han sido someramente señalados. Tal vez podamos avizorar los desafíos que impondrán los cambios de un nuevo siglo a nuestra cultura y, en particular, a nuestro hábitat cercano, urbano y territorial, para actuar de la mejor manera desde nuestras capacidades en aras del bien común. Y sin duda otros colegas, con su disposición heurística, capacidad técnica y mirada crítica contribuirán a la construcción de la cultura del arte arquitectónico en Costa Rica.

\section{Referencias bibliográficas}

Barahona, L. (2000). Arquitectura Contemporánea Costarricense. Reflexiones y proyectos. San José: Grupo Pujol- Martí.

Cuche, D. (1999). La noción de cultura en las ciencias sociales. Buenos Aires:Editorial Nueva Visión.

Fonseca, E. \& Garnier, E. (Eds). (1998). Historia de la Arquitectura en Costa Rica San José: Fundación Museos del Banco Central de Costa Rica.

Grané, J. (1997). Memoria 25 Aniversario Escuela de Arquitectura de la Universidad de Costa Rica.1971-1996. San José: Oficina de Publicaciones de la UCR.

Grané, J. (1998, Julio). La mexicanización de nuestra arquitectura. Imago, (5),37-43. de Costa Rica.1971-1996. San José: Oficina de Publicaciones de la UCR.

Huyssen, A. (2001). En busca del futuro perdido. Cultura y memoria en tiemposde globalización. Buenos Aires: Fondo de Cultura Económica.

Rojas, M. (1993). Historia General de Costa Rica (Tomo V). Madrid: Ediciones FLACSO. 